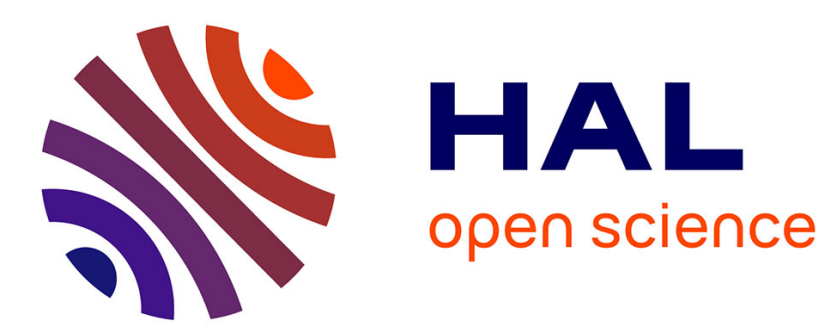

\title{
Être officiel ou faire officiel ? Sur deux styles de barrages routiers en Afrique de l'Ouest (Ghana/ Sénégal)
}

\author{
Sidy Cissokho
}

\section{- To cite this version:}

Sidy Cissokho. Etre officiel ou faire officiel ? Sur deux styles de barrages routiers en Afrique de l'Ouest (Ghana/ Sénégal). Critique Internationale, 2019, Critique internationale, № 83 (2), pp.167189. 10.3917/crii.083.0167 . hal-03543814

\section{HAL Id: hal-03543814 \\ https://hal.univ-lille.fr/hal-03543814}

Submitted on 26 Jan 2022

HAL is a multi-disciplinary open access archive for the deposit and dissemination of scientific research documents, whether they are published or not. The documents may come from teaching and research institutions in France or abroad, or from public or private research centers.
L'archive ouverte pluridisciplinaire $\mathbf{H A L}$, est destinée au dépôt et à la diffusion de documents scientifiques de niveau recherche, publiés ou non, émanant des établissements d'enseignement et de recherche français ou étrangers, des laboratoires publics ou privés. 


\title{
Être officiel ou faire officiel? Sur deux styles de barrages routiers en Afrique de l'Ouest (Ghana/Sénégal)
}

\author{
par Sidy Cissokho
}

L'organisation de contrôles routiers est souvent perçue comme une prérogative fondamentale des autorités administratives ${ }^{1}$. De nombreux travaux ont cependant mentionné 2 , et plus rarement étudié ${ }^{3}$, la façon dont ce type d'activités pouvait être le fruit d'institutions ne disposant pas du label «État» dans les contextes africains. À différents moments de l'histoire du Sénégal et du Ghana, ces pratiques ont par exemple prospéré, et des représentants syndicaux des professionnels du transport ont été associés aux forces de l'ordre dans la gestion de barrages routiers. Les Union Guards au Ghana et les Brigades maraudage au Sénégal ont ainsi acquis le pouvoir d'arrêter et d'infliger des amendes aux chauffeurs professionnels qui ne s'acquittent pas des taxes prélevées par les organisations syndicales au départ des gares routières. En dressant ces barrages routiers, chacune de ces entités exerce ou a exercé une série de contrôles concernant exclusivement les chauffeurs de transports de passagers, et ce parallèlement à l'administration.

Ces pratiques font écho aux débats sur les formes prises par l'État en Afrique. Depuis maintenant plusieurs décennies, des travaux de toutes disciplines se sont appliqués à nuancer les visions les plus communément admises de l'État et de son intervention dans des contextes violents ${ }^{4}$, face à des phénomènes tels que la privatisation ${ }^{5}$, la criminalité $^{6}$, l'insécurité dans les grands centres urbains ${ }^{7}$, ou tout simplement dans le fonctionnement routinier de l'administration ${ }^{8}$. Tous ont questionné, chacun à sa manière, la pertinence de la démarcation stricte entre État et société, le prétendu monopole de l'administration sur un certain nombre d'actions, et exploré les diverses formes de délégation de l'autorité publique en vigueur en Afrique subsaharienne. Tout comme le recours à l'histoire', ce décentrement

\footnotetext{
${ }^{1}$ Ben Bowling, Leanne Weber, «Stop and Search in Global Context: An Overview », Policing and Society. An International Journal of Research and Policy, 21 (4), 2011, p. 353-356.

${ }^{2}$ Nassirou Bako-Arifari, "“Ce n'est pas les papiers qu'on mange !" La corruption dans les transports, la douane et les corps de contrôle », dans Jean-Pierre Olivier de Sardan, Giorgio Blundo, (dir.), État et corruption en Afrique. Une anthropologie comparative des relations entre fonctionnaires et usagers (Bénin, Niger, Sénégal), Paris, Apad/Karthala, 2007, p. 179-225 ; Jean-François Bayart, L'État en Afrique : la politique du ventre, Paris, Fayard, 1989, p. 9-12.

${ }^{3}$ Peer Schouten, Soleil-Perfect Kalessopo, The Politics of Pillage: The Political Economy of Roadblocks in the Central African Republic, Bangui, International Peace Information Service (IPIS)/Danish Institute for International Studies (DIIS), 2017 ; Louisa Lombard, « Navigational Tools for Central African Roadblocks », PoLAR: Political and Legal Anthropology Review, 36 (1), 2013, p. 157-173.

${ }^{4}$ Marielle Debos, « La guerre des préfets. Répression, clientélisme et illégalismes d'État dans l'entre-guerres tchadien », Politix, 104 (4), 2013, p. 47-65.

${ }^{5}$ Béatrice Hibou, La privatisation des États, Paris, Karthala, 1997.

${ }^{6}$ Jean-Francois Bayart, Stephan Ellis, Béatrice Hibou, The Criminalization of the State in Africa, Bloomington, Indiana University Press, 1999.

7 Thomas Kirsch, Tilo Grätz (eds), Domesticating Vigilantism in Africa, Suffolk, Boydell \& Brewer, 2013.

8 Thomas Bierschenk, Jean-Pierre Olivier de Sardan (eds), States at Work: Dynamics of African Bureaucracies, Leyde, Brill, 2014.

${ }^{9}$ Charles Tilly, «La guerre et la construction de l'État en tant que crime organisé », Politix, 13 (49), 2000, p. 97-117.
} 
géographique a contribué à une entreprise plus large de dénaturalisation de l'État au sein des sciences sociales ${ }^{10}$.

Certes, cette littérature ne constitue pas un tout homogène. Elle a cependant mis en évidence la nature ambivalente de l'action d'un nombre impressionnant d'institutions n'ayant pas le label «État», mais disposant de ce qui est généralement perçu comme certains de ses attributs. L'un des enseignements de ces travaux est que la "culture juridique ${ }^{11}$, le 《 statehood $»^{12}$, le « stateness $»^{13}$ et autres manières de faire officiel peuvent prospérer dans des contextes encore considérés par une partie du monde scientifique comme relevant d'une situation d' « État failli » ${ }^{14}$, « fragile ${ }^{15}$ ou $~ «$ faible ${ }^{16}$. De ce point de vue, le manque de moyens matériels de l'administration sur le continent africain n'entame pas plus la puissance des représentations et des symboles associés à l'État qu'elle ne signifie le chaos. Sur la base de ces constatations, les débats sur l'État en Afrique se sont déplacés de la question de sa présence ou de son absence vers l'étude de la multitude des imaginaires qui lui sont associés dans cette partie du monde ${ }^{17}$.

Bien qu'elles ne se situent pas dans un contexte violent ou de faiblesse extrême de l'administration comme la majeure partie des exemples cités dans la littérature susmentionnée, les activités des Union Guards et des Brigades maraudage illustrent parfaitement ces réflexions. Ces deux sortes de barrages sont organisées par des entités privées, représentant un groupe professionnel. Leur légitimité repose cependant sur la mise en scène de leur proximité avec l'idéal type de l'État wébérien, ses fonctionnaires mais aussi et surtout sa symbolique à travers de multiples accessoires. La mise en regard de ces deux cas permet cependant d'aller plus loin que le constat dressé dans certains travaux sur ce type d'institution ${ }^{18}$.

L'action des Union Guards a longtemps été permise par une délégation formelle de l'administration appuyée par le droit. Si elle a doté l'institution ghanéenne de précieuses ressources pour mettre en scène son étaticité, cette particularité l'a aussi rendue plus vulnérable aux évolutions de la conjoncture politique nationale que son homologue sénégalaise. L'action des Brigades est en effet le produit d'une multitude d'accords informels passés avec l'administration à l'échelle des villes ou des régions. Alors que ces

\footnotetext{
${ }^{10}$ La formule de Pierre Bourdieu, «Penser l'État sans la pensée d'État », résume bien l'enjeu et la principale difficulté de ce vaste programme de recherche. Pierre Bourdieu, Sur l'État. Cours au Collège de France (1989-1992), Paris, Le Seuil/Raisons d'agir, 2012.

${ }^{11}$ Jean Comaroff, John L. Comaroff, Law and Disorder in the Postcolony, Chicago, University of Chicago Press, 2006, p. 22-35.

12 Tobias Hagmann, Didier Péclard, Negotiating Statehood: Dynamics of Power and Domination in Africa, Oxford, Wiley-Blackwell, 2011.

${ }^{13}$ Christian Lund, « Twilight Institutions: Public Authority and Local Politics in Africa », Development and Change, 37 (4), 2006, p. 685-705.

${ }^{14}$ Frances Stewart, Graham Brown, Fragile State, CRISE Working Paper N51, Oxford, University of Oxford, 2009.

${ }^{15}$ Robert H. Jackson, Carl G. Rosberg, « Why Africa's Weak States Persist: The Empirical and the Juridical in Statehood », World Politics, 35 (1), 1982, p. 1-24.

16 I. William Zartman, Collapsed States: The Disintegration and Restoration of Legitimate Authority, Boulder, Lynne Rienner, 1995.

${ }^{17}$ Suivant en cela une ancienne piste de recherche ouverte par Philip Abrams dans « Notes on the Difficulty of Studying the State (1977) », Journal of Historical Sociology, 1 (1), 1988, p. 58-89.

18 C. Lund, « Twilight Institutions: Public Authority and Local Politics in Africa », art. cité ; L. Lombard, « Navigational Tools for Central African Roadblocks », art. cité.
} 
deux pratiques ont émergé au même moment dans les années 1980, seuls les barrages sénégalais sont encore opérationnels aujourd'hui.

\section{Une comparaison asymétrique}

Les données concernant le cas sénégalais ont été recueillies lors d'un terrain de type ethnographique mené entre 2009 et 2014 . Elles proviennent tout particulièrement d'une étude menée dans l'une des principales régions du pays, même si des entretiens ont été menés sur cette thématique dans d'autres régions. Elles sont constituées d'une série d'observations directes ainsi que de conversations informelles menées auprès de plusieurs équipes maraudage. Cette partie de mon enquête m'a amené à participer aux activités des Brigades à plusieurs reprises, en tant qu'observateur ou en tant qu'acteur en renseignant les formulaires des amendes délivrées aux chauffeurs en infraction.

Les données concernant le cas ghanéen sont issues des «chutes » d'un terrain de type ethnographique principalement consacré aux pratiques quotidiennes du principal syndicat du transport de passagers. Elles ont été récoltées lors d'un terrain de trois mois mené en 2013 à Accra et dans la ville de Kumasi en région Ashanti. La démarche pratiquée au Sénégal n'a pas pu être appliquée au Ghana, les activités des Union Guards se limitant aujourd'hui au maintien de l'ordre dans les gares routières. J'exploite donc tout particulièrement ici une dizaine d'entretiens semi-directifs effectués avec d'anciens Union Guards, ainsi qu'avec des cadres du syndicat dont cette institution est issue. À cela s'ajoutent les résultats de travaux universitaires très précis sur la période, ainsi que les archives personnelles de certains des enquêtés (photos et textes règlementaires du syndicat). Cette asymétrie peut parfois être considérée comme un biais difficilement surmontable dans les travaux comparatifs ${ }^{19}$. Ici, elle est au contraire au fondement de la comparaison. Parce qu'elle repose sur une série d'arrangements locaux informels, l'existence des Brigades ne peut être documentée qu'à travers la démarche ethnographique. En revanche, celle des Union Guards peut être documentée à travers des sources secondaires parce qu'elle repose sur des arrangements formels.

Le but de la comparaison entre ces deux formes de contrôle n'est pas de distinguer les bonnes manières de faire officiel des mauvaises ${ }^{20}$, mais plutôt de souligner comment, dans ce cas, la forme d'étaticité a priori la plus bricolée est non seulement la moins fragile, mais aussi la plus durable. Je reviendrai tout d'abord sur les trajectoires historiques des Union Guards et des Brigades maraudage pour montrer en quoi chacune de ces institutions est le produit d'une forme d'arrangement singulière. J'insisterai ensuite sur la façon dont ces trajectoires historiques ont donné lieu à deux styles d'étaticité distincte. Enfin, je défendrai l'idée selon laquelle chacun de ces styles est exposé à un type de dénonciation particulier

\footnotetext{
${ }^{19}$ Marie-Hélène Sa Vilas Boas, « Écrire la comparaison lorsque les données sont asymétriques. Une analyse de l'engagement dans les dispositifs participatifs brésiliens », Revue internationale de politique comparée, 19 (1), 2012, p. 61-74.

${ }^{20}$ Pour une stimulante critique comparatiste des approches « tillyennes » de la formation de l'État, voir Kate Meagher, "The Strength of Weak States? Non-State Security Forces and Hybrid Governance in Africa », Development and Change, 43, 2012, p. 1073-1101.
} 
qui donne lieu à des remises en cause radicales dans un cas, et seulement partielles dans l'autre.

\section{Arrangements nationaux et arrangements locaux}

Les Union Guards sont le produit d'une situation révolutionnaire caractérisée par un chevauchement temporaire des univers partisans, administratifs et syndicaux ${ }^{21}$, et théorisée ensuite sous la forme d'une idéologie associant la « société civile » au fonctionnement d'un régime militaire ${ }^{22}$. Les Brigades maraudage sénégalaises sont en revanche issues d'une conjoncture routinière dans laquelle leur capacité à déployer leurs barrages dépend essentiellement de transactions locales, informelles, passées hors du domaine juridique, dans un contexte d'ouverture démocratique.

\begin{tabular}{|c|c|}
\hline Ghana & Sénégal \\
\hline $\begin{array}{l}\text {-1957 : le Ghana devient indépendant. } \\
\text {-1960 : instauration de la première } \\
\text { république. Kwame Nkrumah est président. } \\
\text {-1966 : coup d'État et destitution de } \\
\text { Kwame Nkrumah (sera suivi par les coups } \\
\text { d'État de } 1972 \text { et 1978). } \\
\text {-1979: coup d'État de Jerry Rawlings qui } \\
\text { transmet le pouvoir à Hilla Limann. Début } \\
\text { de la troisième république. } \\
\text {-1981 (31 décembre) : second coup d'État } \\
\text { de Jerry Rawlings et instauration d'un } \\
\text { régime militaire avec ce dernier à sa tête. } \\
\text {-1992: instauration du multipartisme et } \\
\text { élection de Jerry Rawlings, puis début de la } \\
\text { quatrième république. } \\
\text {-2000 : le candidat de l'opposition, John } \\
\text { Kufuor, est élu président. }\end{array}$ & $\begin{array}{l}\text {-1960 : le Sénégal devient indépendant, } \\
\text { Léopold Sédar Senghor est élu président. } \\
\text {-1974 : début de la libéralisation progressive } \\
\text { de la vie politique. } \\
\text {-1981: Léopold Sédar Senghor quitte le } \\
\text { pouvoir et confie le pays à son premier } \\
\text { ministre Abdou Diouf qui est élu président } \\
\text { dans la foulée. } \\
\text {-2000 : première alternance politique et } \\
\text { élection d'Abdoulaye Wade à la présidence de } \\
\text { la République. } \\
\text {-2012: seconde alternance et élection de } \\
\text { Macky Sall à la présidence de la République. } \\
\end{array}$ \\
\hline
\end{tabular}

\footnotetext{
${ }^{21}$ Michel Dobry, Sociologie des crises politiques. La dynamique des mobilisations multisectorielles, Paris, Presses de Sciences Po, 2009.

${ }^{22}$ Paul Nugent, Big Men, Small Boys and Politics in Ghana. Power, Ideology and the Burden of History, 1982-1994, Londres, Pinter, 1995, p. 45-46.
} 


\section{Une intégration par le haut (Ghana)}

Les Union Guards sont directement issus du Ghana Private Road Transport Union (GPRTU). Ce syndicat existe depuis les années 1970 et provient de la fusion de différentes associations locales de chauffeurs, jusque-là uniquement organisées par région ${ }^{23}$. C'est à partir de 1982 et du second coup d'État orchestré par le général Jerry John Rawlings que l'organisation connut un développement sans précédent. Le syndicat et ses démembrements locaux dans les gares - appelés aussi « branches $^{24}$ » - intégrèrent directement la stratégie de maillage du territoire urbain du Provisional National Defence Council, le gouvernement révolutionnaire ${ }^{25}$. Ses liens avec ce dernier permirent au syndicat d'instaurer un quasimonopole sur la représentation des professionnels du transport ${ }^{26}$.

La formation des liens unissant le gouvernement révolutionnaire au mouvement syndical aurait commencé à partir du premier coup d'État mené par J. J. Rawlings, son retrait temporaire et l'élection de Hilla Limann en 1979. Durant cette période, une poignée d'aspirants révolutionnaires infiltrèrent le Trades Union Congress (TUC), ainsi que sa branche transport, le GPRTU. À partir de 1981 et après une campagne interne de dénigrement de la gestion du syndicat, cette poignée d'individus intégra le comité de gestion interne du GPRTU. En 1982, à la suite du second coup d'État et de l'arrivée au pouvoir effective de J. J. Rawlings, leur représentant fut officiellement nommé dirigeant du syndicat ${ }^{27}$.

Ce dernier entreprit alors de réformer le secteur du transport. Depuis les années 1970, la profession de chauffeur et avec elle le secteur du transport souffraient d'une image dégradée dans l'opinion publique ${ }^{28}$. Avec la pénurie d'essence, le transport était l'un des problèmes les plus importants auxquels devait faire face le gouvernement révolutionnaire. L'amélioration de ce secteur offrait une vitrine parfaite à J. J. Rawlings pour illustrer son projet de modernisation de la société. Des formations en gestion et en management encadrées par le gouvernement furent proposées aux dirigeants des cellules locales du syndicat dans les gares. Ces formations allaient de pair avec la création des premiers Workers' Defence Committees, puis des Committees for the Defence of the Revolution durant les premières années qui suivirent le coup d'État.

C'est dans ce contexte qu'une ancienne initiative locale du syndicat fut étendue à l'échelle nationale. Les Union Guards étaient apparus d'abord dans la région Ashanti dans le courant des années 1970. Leur création était l'œuvre d'un syndicaliste, déjà présent à la tête du

\footnotetext{
${ }^{23}$ Michael Stasik, «Vernacular Neoliberalism: How Private Entrepreneurship Runs Public Transport in Ghana », Sociologus, 65 (2), 2015, p. 188-191.

${ }^{24}$ Pour une description détaillée du fonctionnement des gares routières ghanéennes, voir M. Stasik, «Contingent Constellations: African Urban Complexity Seen through the Workings of a Ghanaian Bus Station », Social Dynamics. A Journal of African Studies, 42 (1), 2016, p. 122-142.

${ }^{25}$ Emmanuel Gyimah-Boadi, « Associational Life, Civil Society, and Democratization in Ghana », dans John Willis Harbeson, Donald S. Rothchild, Naomi Chazan (eds), Civil Society and the State in Africa, Londres, Lynne Rienner Publishers, 1994, p. 132-135.

${ }^{26}$ Voir notamment les constatations effectuées au début des années 1990 par P. R. Fouracre, E. A. Kwakye, J. N. Okyere, D. T. Silcock, dans « Public Transport in Ghanaian Cities - A Case of Union Power », Transport Reviews, 14 (1), 1994, p. 45-61.

${ }^{27}$ Entretien avec un ancien dirigeant national du GPRTU de 1982 à 1991, Accra, 6 mars 2013.

${ }^{28}$ Jennifer Hart, Ghana on the Go - African Mobility in the Age of Motor Transportation, Bloomington, Indiana University Press, 2016, p. 121-148.
} 
démembrement régional du syndicat avant le coup de force des partisans de Rawlings au début des années 1980. À leurs débuts, les Union Guards n'étaient pas plus d'une dizaine et servaient de gardes du corps uniquement à ce représentant. Ils étaient entraînés par un militaire à la retraite, devenu le chef d'une des cellules locales du syndicat établie dans l'une des gares de la capitale de la région Ashanti.

À partir de 1983, l'initiative locale fut reprise à l'échelle de tout le pays. L'année officielle de création des Union Guards n'est pas anodine, elle correspond à un mouvement plus large de militarisation des différentes organisations de la société civile soutenant le régime ${ }^{29}$. Ce mouvement était favorisé par le climat de tension qui régnait après une série d'attaques armées contre le régime encore fragile. Le secrétaire général du syndicat informa lui-même la police et l'armée de la création de ce nouveau corps. Il disposa par ailleurs de l'appui de certains membres du gouvernement ${ }^{30}$. L'enjeu était de faire connaître cette création aux différentes strates de l'administration, afin de l'imposer au sein de l'ordre institutionnel établi. Ce souci de publicité n'était pas à sens unique, et il est plus que probable que les Union Guards aient aussi servi au secrétaire général du GPRTU à s'imposer dans l'organigramme révolutionnaire. Ce changement d'échelle accompagna l'extension de la mission des Guards. Ceux-ci n'étaient plus simplement des gardes du corps des cadres du syndicat, ils étaient également les garants du maintien de l'ordre dans les gares routières. L'objectif de départ était de choisir cinq personnes par destination parmi les travailleurs en charge de l'inscription des véhicules et de leur chargement au sein des gares, puis de leur donner une formation de trois mois. Une relation du secrétaire général du GPRTU et de J. J. Rawlings, surnommée « Flashman », fut nommée à la tête des Guards à partir de $1986^{31}$. Tailleur d'origine, «Flashman » n'avait alors aucun lien avec le monde du transport. Il était un pur produit de la révolution. Après avoir été un membre actif des Comités de défense de la révolution et avoir participé au coup d'État de 1982, il avait fait partie des militants qui avaient bénéficié d'un entraînement militaire en Allemagne de l'Est entre 1982 et son arrivée à la tête des Guards en $1986^{32}$.

En 1988, 1989 et 1990, les conditions de cette délégation furent précisées et étendues par une série de circulaires stipulant explicitement que le «GPRTU [était] reconnu par le gouvernement comme la seule organisation contrôlant, régulant le mouvement et les opérations de tous les véhicules au sein des gares routières $»^{33}$. Le syndicat devint 1 ' «agent légal du gouvernement pour recouvrir les taxes quotidiennes pour tous les véhicules commerciaux $\gg{ }^{34}$. Les pouvoirs qui lui étaient conférés par ces documents lui permirent de

\footnotetext{
${ }^{29}$ P. Nugent, Big Men, Small Boys and Politics in Ghana. Power, Ideology and the Burden of History, 19821994, op. cit., notamment p. 119.

${ }^{30}$ Notamment d'Ebo Tawiah, alors membre influent du Provisional National Defence Council. Entretien avec « Flashman », ancien dirigeant national des Union Guards, Accra, 14 mars 2013.

${ }^{31}$ L'arrivée de « Flashman » à la tête des Union Guards correspond à un remplacement plus large d'une partie des cadres dirigeants des organes de la révolution par des personnes pourvues d'une formation militaire. $\mathrm{P}$. Nugent, Big Men, Small Boys and Politics in Ghana. Power, Ideology and the Burden of History, 1982-1994, op. cit., p. 128.

${ }^{32}$ L'envoi de civils en formation militaire à Cuba, en Allemagne et en Libye correspond à une tentative du régime révolutionnaire de se doter de ses propres cadres militaires après les nombreux soubresauts auxquels il a été exposé durant ses premières années. P. Nugent, Big Men, Small Boys and Politics in Ghana. Power, Ideology and the Burden of History, 1982-1994, op. cit., p. 122.

${ }^{33}$ Circulaire émise par le ministre de l'Administration locale et adressée à l'ensemble de l'administration, ainsi qu'aux dirigeants des autres syndicats du secteur du transport, 1989. Archives privées du GPRTU. ${ }^{34}$ Ibid.
} 
coupler la récolte de ses propres taxes par les différentes branches dans les gares à celle des taxes de l'État. Le syndicat reversait ensuite la part de l'État aux assemblées métropolitaines qui restaient propriétaires des gares. L'organisation gardait cependant sur cette part une commission qui lui servait, entre autres, à rémunérer une partie des Union Guards.

En 1992, dans un contexte de libéralisation politique, le statut juridique des Guards fut renforcé une dernière fois, non plus par une simple circulaire mais par une loi. Celle-ci attribuait aux Guards l'autorité nécessaire pour intervenir sur la route, et non plus seulement dans les gares. Elle étendait leur domaine d'activités à la répression d'un certain nombre de délits routiers sur l'ensemble de la population conduisant un véhicule commercial. Pour ce faire, les Guards furent placés sous la double tutelle du syndicat et de la police. À leur apogée ils représentaient un contingent d'environ 200 individus pour la seule région d'Accra ${ }^{35}$.

\section{Une intégration par le bas (Sénégal)}

Au Sénégal, les Brigades maraudage sont, elles aussi, un sous-produit de l'activité syndicale. Ce sont en effet des émanations du Syndicat national des travailleurs des transports routiers du Sénégal, la branche transport de la Confédération nationale des travailleurs du Sénégal. L'histoire des Brigades est en revanche marquée par un rapport au pouvoir largement plus décentralisé que dans le cas ghanéen.

Durant les années 1970, les chauffeurs étaient représentés par les organisations des transporteurs, c'est-à-dire des propriétaires de véhicules. Selon les entretiens menés avec certains des protagonistes de l'époque, une distanciation s'opéra entre les deux groupes à la fin des années 1980, notamment parce que les chauffeurs jugeaient les transporteurs qui les représentaient trop proches du pouvoir central et donc éloignés de leurs réalités quotidiennes. Dès lors, les anciennes associations mutualistes rassemblant les chauffeurs à l'échelle des gares furent investies de nouvelles significations. Elles n'étaient plus seulement des associations d'entraide mais aussi, et peut-être même d'abord, des associations représentant la profession de chauffeur.

À différents moments et sur l'ensemble du territoire ${ }^{36}$, les dirigeants de ces associations, aussi appelées « regroupements », prirent le contrôle des gares routières des mains des représentants des transporteurs, parfois de façon violente. Ils s'emparèrent du même coup du système de perception auparavant mis en place par les transporteurs. La collecte s'effectuait désormais toujours auprès des chauffeurs, mais aussi au nom des chauffeurs et pour les chauffeurs. Cette rhétorique transforma dès lors le «maraudage »-1'embarquement de passagers à l'extérieur des gares - en un délit grave au sein de la profession, car contrevenant à ses principes d'entraide.

Tout au long des années 1990, les nombreux dirigeants des regroupements s'imposèrent comme de véritables «notables ${ }^{37}$. Ils s'intégrèrent dans des configurations mêlant les représentants de l'administration et des partis politiques, similaires à celles dans lesquelles les

\footnotetext{
${ }^{35}$ Je ne dispose pas d'estimations pour les autres régions ou à l'échelle nationale.

${ }^{36}$ Le caractère national de ces arrangements locaux peut s'expliquer par la nature des lieux dans lesquels ils se sont concrétisés : les gares routières. La diffusion de ce mouvement a probablement été d'abord assurée par la circulation des chauffeurs.

${ }_{37}$ Pierre Grémion, Le pouvoir périphérique - Bureaucrates et notables dans le système politique français, Paris, Le Seuil, 1976, p. 154-171.
} 
grands transporteurs qui les précédaient été intégrés. Cependant, cette intégration s'effectuait d'abord à l'échelle des villes ou des régions et non plus à celle de la nation. Elle passait par des pratiques d'intermédiation politique entre les chauffeurs des gares et les relais locaux de figures politiques nationales, notamment lors des périodes électorales. Elle passait également par des pratiques d'intermédiation administrative entre ces mêmes chauffeurs et l'administration déconcentrée dans des affaires allant du retrait de permis au changement d'immatriculation, en passant par des procès pour accident.

Toujours durant les années 1990, ce tissu associatif intégra la branche transport de la Confédération nationale des travailleurs du Sénégal, notamment grâce à l'action du secrétaire général du Syndicat national des travailleurs des transports routiers du Sénégal qui mit en œuvre une stratégie systématique visant à rallier les représentants locaux des chauffeurs. Durant cette décennie, ceux-ci apparurent sur la scène nationale, et nouèrent des contacts avec le pouvoir central, principalement à travers des épisodes de grève paralysant l'ensemble du territoire. À la différence du GPRTU ghanéen durant la même période, les échanges du syndicat sénégalais avec le pouvoir central furent donc fondés sur un rapport de force.

C'est dans ce contexte historique précis qu'il faut replacer la lutte contre le maraudage. L'activité des Brigades ne repose pas sur une loi élaborée en haut lieu comme dans le cas des Union Guards, mais sur une série de collusions locales entre les représentants de l'administration et ceux des chauffeurs. Certes, la lutte contre le maraudage concerne systématiquement le trafic interurbain, mais la périodicité de l'implantation des Brigades le long des routes ou encore la forme que peuvent prendre les barrages varient, sur l'ensemble $\mathrm{du}$ territoire, en fonction des regroupements et de la plus ou moins grande présence de l'administration dans la ville ou dans la région où ils officient.

Le fait que le statut des Brigades ne soit pas directement entériné par la loi ne veut pas dire que la lutte contre le maraudage n'entretient aucun rapport avec ce registre normatif. Cependant, au lieu de s'appuyer directement sur la norme juridique, une partie du processus de légitimation des activités des Brigades repose sur la capacité du registre normatif professionnel des regroupements à utiliser les failles pratiques du registre juridique dans le cadre de négociations locales avec les représentants de l'administration. Dans la loi, le maraudage relèverait des «infractions aux règles concernant l'usage des voies publiques ouvertes à la circulation $»^{38}$. Dans le décret d'application de cette même loi, la maraude relèverait des infractions relatives au stationnement ${ }^{39}$. Dès lors, même s'ils font respecter une norme propre à la profession des chauffeurs, les membres des Brigades maraudage sanctionnent de fait une infraction à la loi, comme certains de leurs représentants aiment à le rappeler. À cette proximité viennent s'ajouter des difficultés relatives à la nature même de l'infraction. Le mauvais usage des voies publiques ouvertes à la circulation est une infraction difficile à sanctionner et seuls les regroupements disposent des effectifs nécessaires pour effectuer ce travail. L'embarquement de passagers peut en effet se faire n'importe où sur la route, en quelques minutes seulement, et il est d'autant plus difficile à constater que les gendarmes et les policiers fonctionnent déjà en sous-effectifs.

\footnotetext{
${ }^{38}$ Loi n ${ }^{\circ}$ 2002-30 portant sur le code de la route au Sénégal, adoptée par l'Assemblée nationale le jeudi 12 décembre 2002, Titre III.

${ }^{39}$ Décret n$^{\circ}$ 2004-13 du 19 janvier 2004, fixant les règles d'application de la loi n 2002-30.
} 
Dans la région où j'ai mené mon enquête, cette situation peut inciter certains agents à opérer une classification parmi les infractions et à les hiérarchiser. Alors qu'il est au cœur des normes encadrant la profession de chauffeur, le maraudage est perçu comme une infraction mineure par les agents administratifs en charge de faire respecter la loi. Pour cette infraction et une série d'autres, les forces de l'ordre préfèrent « faire de la prévention », c'est-à-dire laisser repartir le véhicule sans le verbaliser, quelquefois en échange d'une faible somme d'argent. Le fait que les infractions liées au stationnement soient délaissées n'est cependant pas uniquement le produit d'arbitrages purement symboliques de la part des fonctionnaires, il est aussi le fruit d'arbitrages économiques. Premièrement, pour les gendarmes et les policiers, il est des infractions et des groupes de contrevenants plus rémunérateurs que d'autres. Sur l'axe Dakar-Bamako par exemple, les policiers et les gendarmes présents sur le bord des routes préfèrent se concentrer sur les gros porteurs auxquels ils peuvent systématiquement extorquer de l'argent plutôt que sur les voitures de transport de passagers. Deuxièmement, et bien que cette pratique soit difficilement avouée, le produit de chaque amende maraudage est partagé entre les représentants de l'administration et ceux de la profession, suivant un ratio différent en fonction des localités.

La forme prise par les accords permettant aux Guards et aux Brigades d'effectuer des contrôles sur la population des chauffeurs de transport en commun dans l'espace public est sensiblement différente. Dans le cas ghanéen, il s'agit d'accords directement issus du contexte révolutionnaire et cristallisés par le droit, relativement uniformes sur l'ensemble du territoire national. Dans le cas sénégalais, il s'agit d'accords conclus en marge de la loi à l'échelle locale, dans un contexte historique de prise de distance entre le pouvoir central et le mouvement syndical ${ }^{40}$. La présence des Brigades maraudage sur le bord des routes est le produit d'accords variables donnant lieu à un partage du travail répressif entre fonctionnaires et représentants des chauffeurs dans les villes et leurs alentours. Ces différents modes d'intégration au pouvoir central ne sont pas sans conséquence. Ils déterminent les ressources dont disposent les Guards et les Brigades pour s'imposer dans l'espace public

\section{Deux styles d'étaticité}

Pour légitimer leurs actions sur le bord des routes, les Guards et les Brigades jouent sur le paraître administratif. Les rapports différents que chacune de ces institutions entretient avec le pouvoir central ne leur confèrent cependant pas les mêmes ressources pour effectuer cette mise en scène. Au Ghana, la relation privilégiée entretenue par le syndicat avec le gouvernement révolutionnaire donna aux Guards la possibilité de se constituer en un corps à part entière. Leur légitimité reposait en grande partie sur la mise en avant de signes faisant directement référence à l'armée. Au-delà des signes, une formation d'inspiration militaire longue de plusieurs mois permettait l'incorporation d'un ethos bureaucratique. Au Sénégal, bien que la légitimité de leur présence sur le bord des routes repose sur les mêmes prétentions bureaucratiques, les Brigades disposent d'un panel d'attributs beaucoup moins puissant pour alimenter cette croyance chez l'usager. La légitimité de leur action paraît bricolée,

\footnotetext{
${ }^{40}$ Momar Coumba Diop, Mamadou Diouf, Le Sénégal sous Abdou Diouf. État et société, Paris, Karthala, 1990, p. 223-250.
} 
essentiellement fondée sur l'entretien d'une proximité physique avec les représentants de l'État, ainsi que sur l'usage de l'écrit.

\section{Étaticité incorporée (Ghana)}

Le premier élément venant conférer aux Union Guards tous les attributs d'un «corps habillé $»^{41}$ et donc distinct du reste de la profession est leur recrutement. À leur apogée, durant les années 1990, celui-ci s'effectuait selon deux canaux. Une partie des Guards était recrutée et rémunérée directement par les cellules du syndicat présentes dans les gares, les branches. Ces entités géraient les départs pour les différentes destinations au sein des gares routières. Leurs représentants assuraient la collecte d'argent auprès des chauffeurs pour le compte du syndicat et pour celui de l'État. Les branches reversaient ensuite une partie de ces revenus à l'échelon national du syndicat qui versait à son tour sa part au Trésor public. Les activités de cette partie du contingent des Guards étaient divisées entre les gares et les routes. Une autre partie des Guards, considérée comme hiérarchiquement supérieure à la précédente, était recrutée directement par l'échelon national du syndicat, rémunérée par celui-ci sur la base des revenus versés par les branches. Leur mission se concentrait sur les contrôles routiers ${ }^{42}$.

Pour les branches, recruter plusieurs Union Guards était un signe de prospérité. Compte tenu de la dureté réputée de leur entraînement, c'était également la garantie incontestable d'avoir un personnel de qualité. L'autorité des Guards était l'une des plus élevées dans la hiérarchie du garage ${ }^{43}$ et leur influence s'étendait sur l'ensemble de la gare et non sur le seul espace contrôlé par les branches. Le prestige et le respect qui leur étaient attachés suscitèrent une âpre compétition entre les branches qui rivalisaient d'ardeur pour accroître le nombre de leurs recrutements. Cette rivalité valait aussi pour l'échelon national du syndicat qui, soucieux de préserver son ascendant sur ses démembrements locaux, accroissait régulièrement le nombre de ses recrus.

Cette course ouvrit la voie à un autre type de recrutement, majoritaire et extérieur aux réseaux du monde du transport car passant par les connaissances directes ou indirectes des cadres nationaux du syndicat. Cette seconde voie de recrutement explique notamment la présence de femmes au sein des Guards, alors que la profession de chauffeur et les équipes de gestion des départs au sein des gares sont exclusivement masculines. C'est dans ce cadre que, dans la région d'Accra, au moins une dizaine d'individus provenant directement des Comités de défense de la révolution et ayant bénéficié d'une formation militaire à l'étranger (Allemagne de l'Est ou Libye) intégrèrent le corps des Guards ${ }^{44}$.

D'autres facteurs permirent aux Guards de s'autonomiser partiellement de la profession de

\footnotetext{
${ }^{41}$ Marielle Debos, Joël Glasman, «Politique des corps habillés. État, pouvoir et métiers de l'ordre en Afrique », Politique africaine, 128 (4), 2012, p. 5-23.

42 Je me base ici sur la constitution du syndicat de 1995, spécifiant le fonctionnement théorique de l'organisation.

${ }^{43}$ Le mot « garage » est employé ici comme un synonyme de « gare routière », de la même façon que dans le langage courant au Sénégal.

${ }^{44}$ Les dates de ces séjours d'entraînement sont différentes selon les témoignages. Certains évoquent la période située entre la sortie de prison du général Rawlings après son premier coup d'État en 1979 et son arrivée effective au pouvoir à la toute fin de l'année 1981, d'autres mentionnent plutôt le milieu des années 1980, au moment où le régime était installé.
} 
chauffeur. Tout d'abord, l'uniforme (photo 1). Sa couleur bleue, qui rappelait directement celui de la police, a valu aux Guards le surnom de «Blue-Blue ». Cependant, une grande partie de la symbolique de cet uniforme fut empruntée à l'armée, de loin la plus puissante à l'époque, du fait des différents coups d'État menés par ses représentants. Les chaussures, la ceinture, le béret ou encore les épaulettes étaient autant d'éléments qui renvoyaient à cette institution et à ses codes, en l'occurrence vestimentaires. Autres détails, sur la manche droite de sa chemise, chaque Guard affichait son grade suivant une dénomination, là encore, proche de celle de l'armée. Une lanière distinguait par ailleurs les membres appartenant à l'échelon national du syndicat de ceux appartenant à l'une de ses branches.

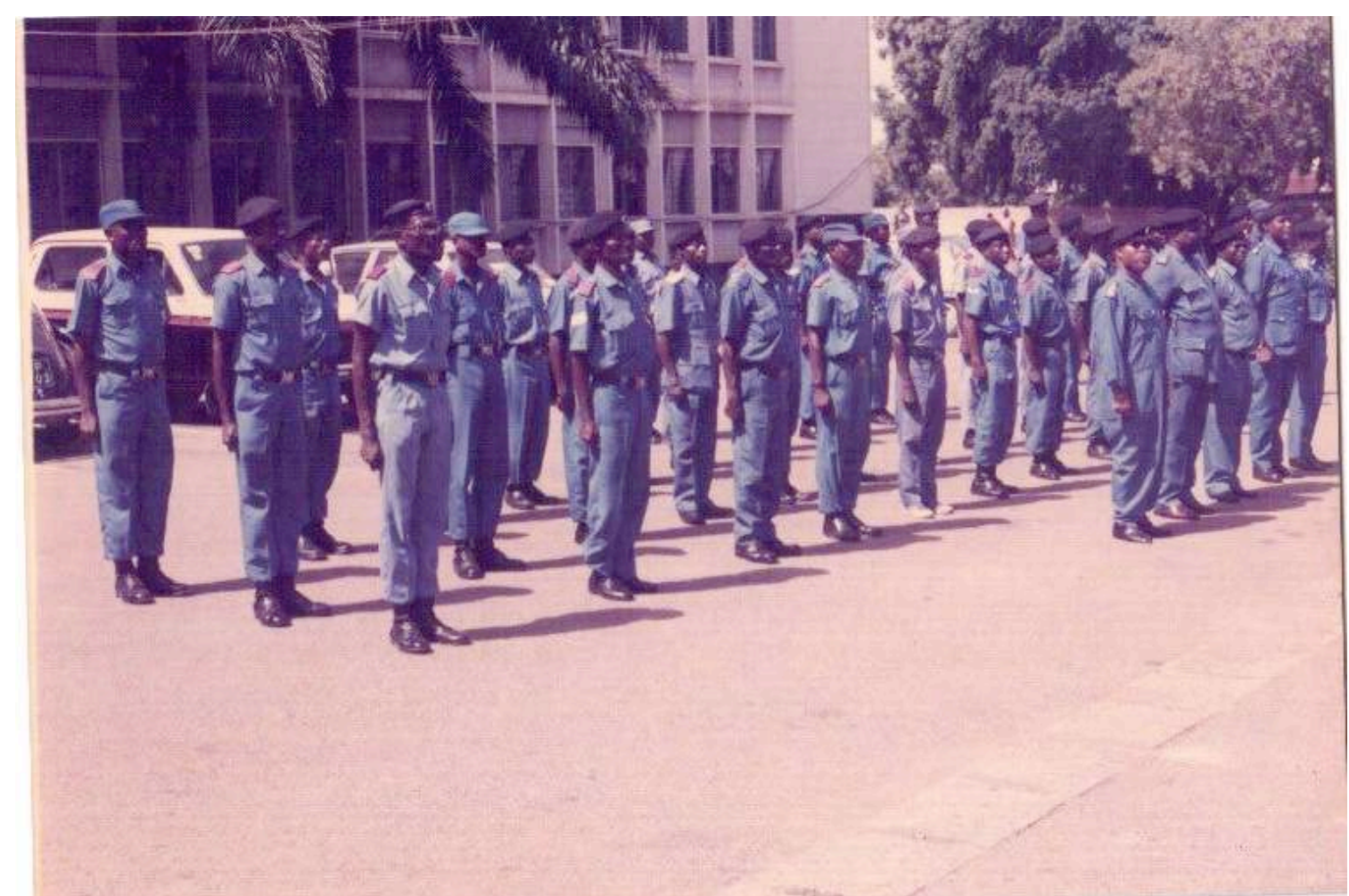

Source : Archives privée d'un ancien formateur des Guards, recueillie à Accra en mars 2013.

L'association des Union Guards aux autres corps de l'administration en uniforme lors des cérémonies officielles prolongeait le mimétisme avec les «corps habillés» de l'administration. Elle contribuait en outre au travail continu de promotion de l'institution entamé dès les premières notes signifiant la création du corps aux différents échelons de l'administration au début des années 1980 (photo 2). Ce jeu d'imitation avait cependant ses limites puisque, contrairement aux représentants de la police ou de l'armée, les Guards ne disposaient pas du droit de port d'armes à feu. 


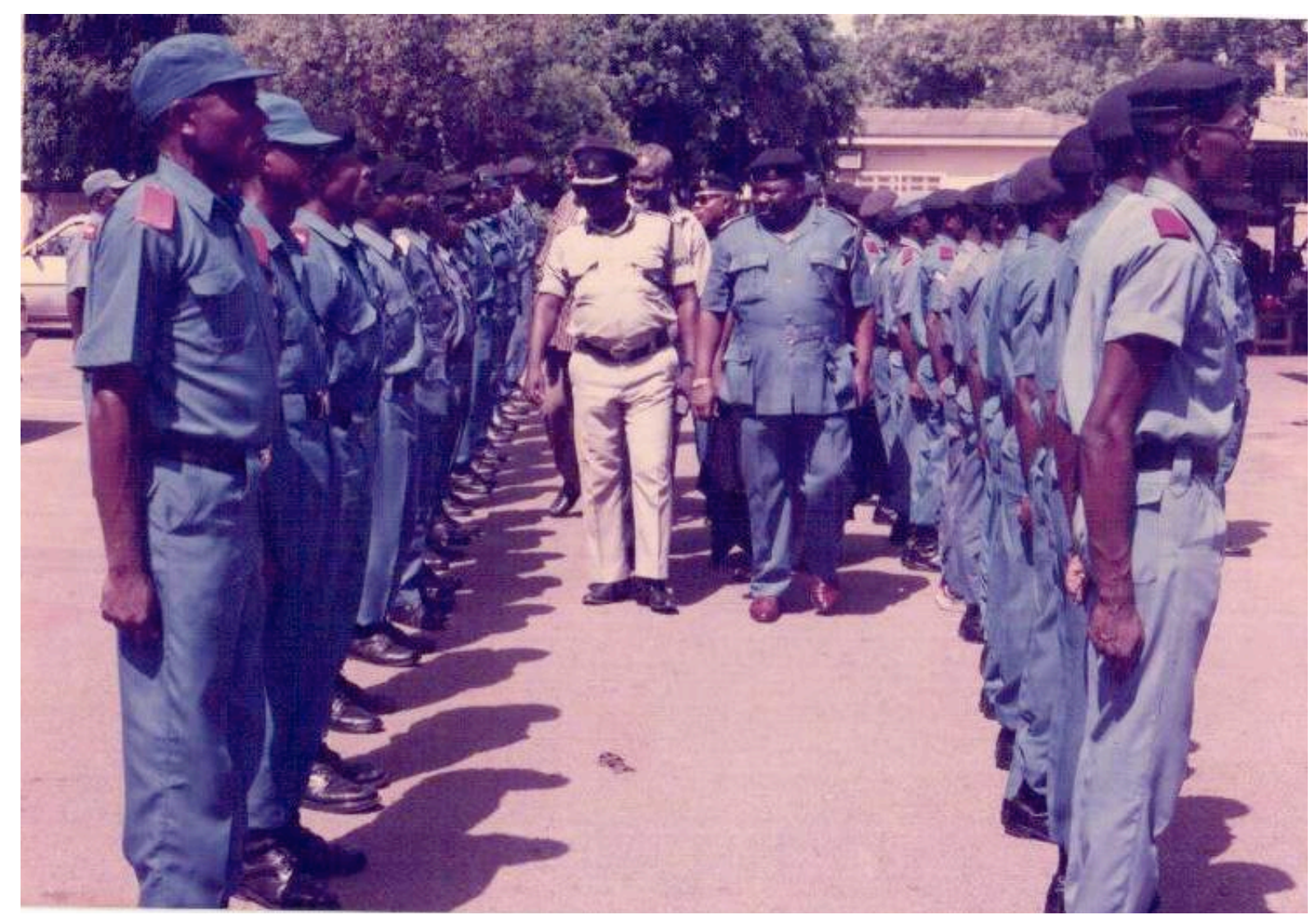

Source : Archives privée d'un ancien formateur des Guards, recueillie à Accra en mars 2013.

Lors du recrutement, le contingent de chaque promotion était envoyé en formation pour une durée de six à neuf mois selon les récits et les époques ${ }^{45}$. La formation s'effectuait dans les locaux de l'armée et sous le contrôle conjoint d'un officier et d'un gradé du corps des Union Guards (photo 3). Les récits recueillis désignent ce moment comme une étape importante dans la création d'un véritable «esprit de corps ». La difficulté de l'épreuve provoquait de nombreux départs et marquait, pour ceux qui restaient, une véritable rupture avec leur ancienne vie. Au-delà des exercices physiques, les recrues réapprenaient à se tenir, à marcher. On leur enseignait les valeurs associées à leurs nouvelles fonctions : le respect de l'uniforme et le devoir, présentés comme les piliers de la supériorité des Guards par rapport aux autres membres de base du syndicat.

\footnotetext{
${ }^{45}$ Dans les quelques pages qu'il consacre aux relations entre le GPRTU et le gouvernement révolutionnaire durant les années 1980 et 1990, E. Gyimah-Boadi mentionne l'existence de Guards, mais ne cite qu'une formation « courte ». E. Gyimah-Boadi, « Associational Life, Civil Society, and Democratization in Ghana », cité, p. 134.
} 


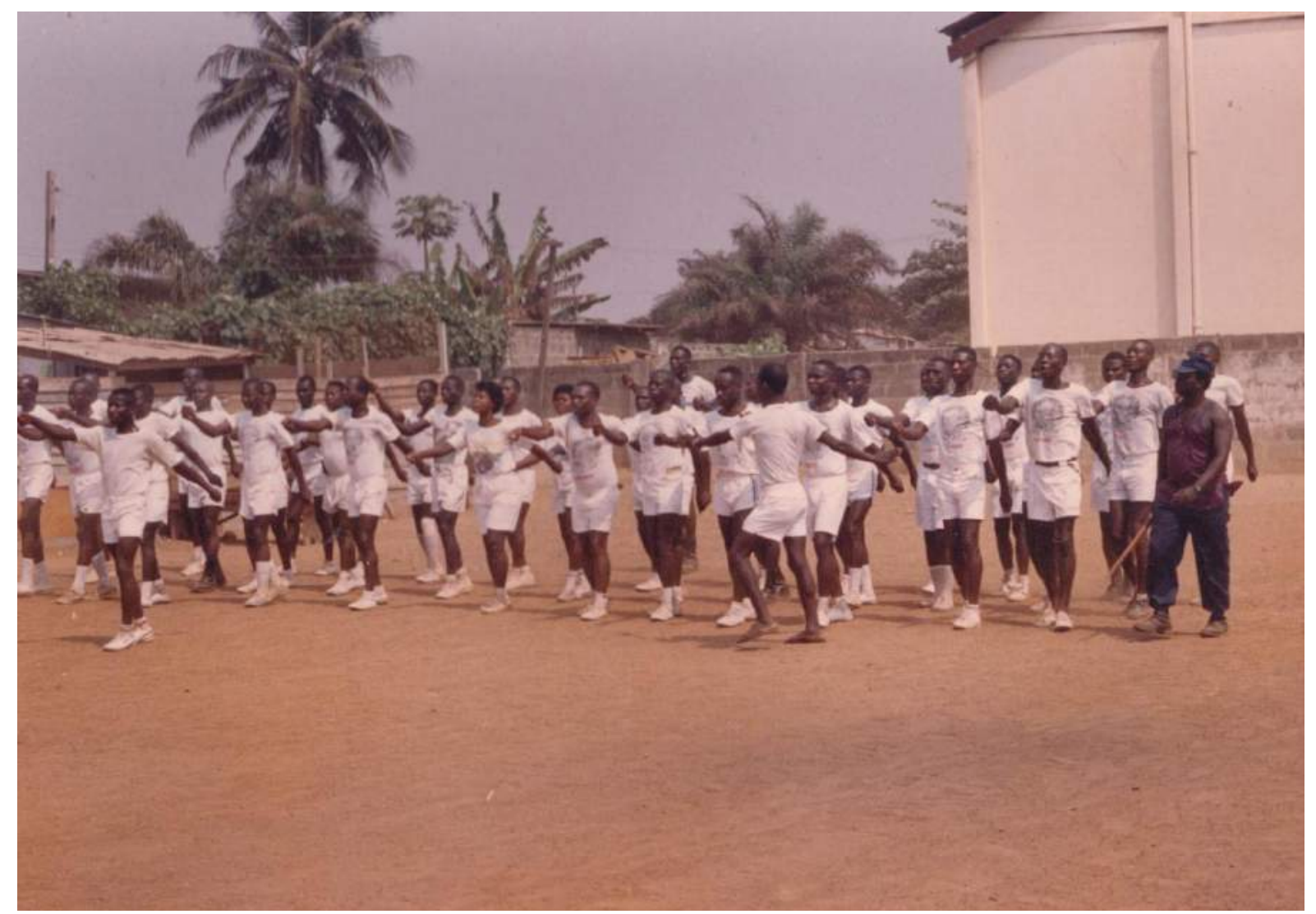

Source : Archives privée d'un ancien formateur des Guards, recueillie à Accra en mars 2013.

\section{Étaticité bricolée (Sénégal)}

À l'incorporation de l'ethos bureaucratique chez les Guards répond une certaine forme d'amateurisme chez les Brigades maraudage. Le nombre de personnes par Brigade, ainsi que la fréquence de leurs activités varient en fonction des garages. Dans les capitales régionales où s'est déroulée mon enquête, les Brigades se composent de deux équipes formées de deux ou trois personnes affectées à plein temps à la répression du maraudage et se relayant aux entrées et aux sorties de la ville jour et nuit. Dans les villes secondaires, leur activité est réduite aux jours de marché. Les membres des équipes maraudage partagent alors leur temps entre les activités sur le bord des routes et celles dans la gare en tant que membres du bureau exécutif du regroupement ou bien comme rabatteurs de clients.

Dans chacun de ces cas, les membres des Brigades sont d'anciens chauffeurs. Ils sont systématiquement rattachés et payés par les regroupements, sur la base de la collecte effectuée dans les gares auprès des chauffeurs. Sur le plan des apparences, aucun uniforme ne les distingue a priori d'un chauffeur lambda. Le processus de différenciation à l'œuvre pour les Guards est donc loin d'être aussi abouti pour les Brigades. Cette situation ne signifie pas pour autant que celles-ci ne tentent pas de mettre en conformité leurs actions avec l'idéal et l'esthétique bureaucratiques ${ }^{46}$. Pour ce faire, elles ont recours à une série d'accessoires renvoyant aux attributs de l'État et conférant à leurs activités toutes les apparences de la légalité. La légitimité des Brigades à opérer sur les routes est cependant

\footnotetext{
${ }^{46}$ Mirco Göpfert, «Bureaucratic Aesthetics: Report Writing in the Nigérien Gendarmerie », American ethnologist, 40 (2), 2013, p. 324-334.
} 
fondée sur l'entretien d'une confusion entre leurs activités et celles d'autres institutions disposant effectivement du label «État », plutôt que sur la réelle possession de ce label et l'incorporation des façons d'être qui lui sont associées.

Un membre d'une Brigade maraudage décrit ainsi sa stratégie face aux gendarmes: «Quand ils arrivent, on prend une voiture et on les suit, on mange au même moment qu'eux et on descend quand ils descendent $\gg^{47}$, propos corroborés par mon expérience au sein des Brigades. Conscient de cette stratégie, un gendarme prévient : « Les gens du regroupement sont malins, ils viennent se coller à nous $\gg{ }^{48}$. Prendre les attributs de l'État, c'est d'abord installer un décor dans lequel la présence de représentants de l'administration qui, eux, disposent effectivement de ce label ont une place importante. Si la cohabitation entre employés municipaux et représentants des chauffeurs n'est que fortuite dans des lieux tels que les postes de perception au sein des gares routières, celle des équipes maraudage avec les forces de l'ordre sur le bord des routes est le fruit d'une véritable recherche, comme pour leurs locaux et leurs véhicules en poste à la sortie des villes. Cette recherche est d'abord motivée par le souci des Brigades de préserver l'apparence légale de leur action. Le décret portant sur le code de la route précise en effet que seul « un agent agréé par le ministère des Transports terrestres » peut immobiliser un véhicule ${ }^{49}$. Selon mes observations, lorsqu'un véhicule est arrêté pour maraudage, c'est donc toujours en collaboration directe avec le policier ou le gendarme présent. Les membres de la Brigade reconnaissent eux-mêmes qu'en tant que « civils » ils n'ont pas le droit de confisquer son permis à un chauffeur.

L'autre élément conférant à l'activité des Brigades maraudage certains des attributs étatiques est l'écrit. Dans les garages où mon enquête s'est concentrée, la lutte contre le maraudage ne se joue pas uniquement au niveau des postes maraudage, mais aussi en ville, grâce à une série d'espions placés à des endroits stratégiques où les véhicules de transport en commun sont susceptibles d'embarquer des passagers en dehors du contrôle des regroupements. Ces espions sont chargés de noter l'heure, l'endroit, le numéro de la plaque d'immatriculation, et de détailler le plus possible l'infraction, exercice qui peut parfois être problématique au sein d'une population pour une large part non alphabétisée en français.

L'ensemble de ces informations est ensuite reporté dans un ou plusieurs livres en fonction d'un classement par région et/ou par type de véhicule. Une partie du temps passé au niveau des postes maraudage peut être employée à mémoriser les numéros de plaques listées dans le livre, et chaque passage de véhicule suspect y fait l'objet d'une vérification ${ }^{50}$. Les infractions ainsi consignées peuvent remonter jusqu'à quatre ans. Cependant, l'efficacité de cette recherche repose aussi sur une connaissance intime du parc de véhicules acquise par les membres des Brigades durant leur carrière en tant que chauffeur, souvent sur le même itinéraire, le long de l'une des sept routes nationales traversant le pays. Cette connaissance leur permet d'associer à la silhouette et à la couleur d'un véhicule l'identité d'un chauffeur, voire une plaque d'immatriculation.

\footnotetext{
${ }^{47}$ Entretien, secrétaire général du regroupement, 19/04/11.

${ }^{48}$ Entretien, gendarme à la retraite, 30/07/12.

${ }^{49}$ Décret n$^{\circ}$ 2004-13 du 19 janvier 2004, article 111.

${ }^{50}$ Statistiquement, les véhicules contrevenants sont pour la plupart des cars, car ils roulent le plus souvent à moitié pleins et sont donc tentés de prendre des passagers en ville.
} 
L'ensemble des garages dispose par ailleurs de carnets d'amendes à leur nom servant à enregistrer le motif de l'infraction, la plaque du véhicule concerné, le montant de l'amende, les signatures du contrevenant et de celui qui la délivre ${ }^{51}$. Le feuillet original est remis au conducteur, le double carbone, qui sert de preuve à l'équipe maraudage, est destiné aux autres membres du regroupement. Le respect de la part de ces derniers des règles de fonctionnement administratives répond à la nécessité de rendre compte de leurs pratiques non seulement aux contrevenants, mais aussi à ceux qu'ils représentent.

Enfin, dans la répression du maraudage, les membres des Brigades insistent sur l'insertion des regroupements de chauffeurs dont ils sont issus dans le dispositif administratif local, ainsi que sur la bonne structuration de ces associations. De leur point de vue, ils agissent d'abord en qualité de représentants du président élu de la gare routière et d'associations qui sont pourvues d'un organigramme indiquant une répartition précise des tâches, d'un règlement intérieur ainsi que d'un récépissé émanant du ministère de l'Intérieur, autant de références à l'univers bureaucratique.

À un mode d'intégration au pouvoir central renvoient un certain type de ressources pour s'imposer en tant qu'autorité légitime sur le bord des routes. Les liens entretenus par le syndicat du secteur du transport ghanéen avec le gouvernement révolutionnaire et le rapport à la loi qui en découlait garantissaient aux Guards leur pleine et entière intégration aux réseaux d'institutions composant l'administration à l'échelle nationale. Cette intégration fit d'eux les détenteurs d'un certain nombre d'attributs bureaucratiques, au premier rang desquels se trouvait l'uniforme. La présence légitime des Brigades sur les routes est, elle aussi, le fruit d'une proximité savamment entretenue avec l'idéal administratif, mais cette fois sans aucun encadrement par la loi. Cette situation fait donc reposer la mise en scène des Brigades non pas sur l'incarnation de l'idéal bureaucratique mais sur une série de tactiques mobilisant des décors et des accessoires censés le véhiculer.

\section{Deux formes de vulnérabilité}

On pourrait s'interroger sur la réelle pertinence de cette opposition entre la mise en scène d'une étaticité « incorporée » et celle d'une autre «bricolée ${ }^{52}$. Après tout, Brigades et Guards occupent tous deux le bord des routes et y contrôlent la population des chauffeurs de transport en commun. Tous deux fondent de plus leur légitimité sur une série d'attributs puisant dans le même imaginaire bureaucratique, même si, pour des raisons historiques, les uns empruntent plus spécifiquement au registre militaire. Cette différence de ressources a cependant un impact sur le rapport à l'usager de chacune de ces institutions. Plus encore, ces différents styles de mise en scène n'ont pas la même longévité. Au moment de mon enquête, il y avait des Brigades maraudage le long des routes, tandis que l'action des Union Guards se cantonnait au seul espace des gares routières. La comparaison permet alors d'avancer une idée contre-intuitive. La faiblesse apparente de l'étaticité bricolée des Brigades fait de celles-ci une institution plus stable dans le temps que les Union Guards.

\footnotetext{
${ }^{5 l}$ En imitation du modèle du carnet fixé par un arrêté conjoint des ministres des Transports, des Forces armées, de l'Intérieur et des Finances. Voir article 146 de la loi n² 2002-30.

${ }^{52}$ Michel-Rolph Trouillot, « The Anthropology of the State in the Age of Globalization: Close Encounters of the Deceptive Kind », Current Anthropology, 42 (1), 2001, p. 25-138.
} 


\section{L'agonie d'une institution (Ghana)}

Une première remarque s'impose. On aurait tort d'accorder trop de crédit au discours et à la symbolique qui entouraient l'institution des Union Guards. Certes, ce discours était efficace et contribuait à la légitimation de leurs actions, mais leur style d'étaticité ne faisait pas automatiquement correspondre leurs pratiques à l'idéal bureaucratique wébérien. Dans les faits, les Guards étaient soumis aux mêmes tensions et autres doubles injonctions que l'ensemble du personnel administratif au contact de l'usager ${ }^{53}$. Bien que n'ayant pas valeur d'observation, les entretiens que j'ai réalisés avec d'anciens Guards mettent en évidence la situation ambivalente créée par la proximité entre d'anciens chauffeurs devenus Guards et d'autres toujours en activité, pour la partie du contingent recrutée directement parmi les travailleurs des gares routières. Pour éviter les éventuelles collusions entre Guards et chauffeurs, les équipes employées par l'échelon national du syndicat étaient souvent appelées en renfort sur de courtes périodes et à travers tout le pays. Cet échelon était supposé être dégagé de toutes les sociabilités locales pouvant être nouées par les Guards provenant des « branches » en contact quotidien avec les chauffeurs dans les gares.

Malgré ces précautions, il semble que, en dehors du travail de perception et de vérification du paiement des taxes, seule une toute petite partie des conflits inhérents à la répression des infractions routières aboutissait devant la police. Le plus souvent, les Guards préféraient emmener le contrevenant directement devant les cadres du syndicat. Sans qu'il soit réellement possible d'évaluer l'ampleur de ces pratiques, il semble que, dans un certain nombre de cas, les Guards aient d'abord servi de médiateurs entre la population des chauffeurs commerciaux et les forces de l'ordre plutôt que de réels substituts à ces dernières.

Seconde remarque. Il semble que la symbolique et le discours entourant l'institution des Union Guards aient rendu celle-ci fragile, car dépendante de la conjoncture politique. Au cours des années 1990, dans le prolongement de la période révolutionnaire, le corps des Union Guards augmenta en taille et s'étendit sur l'ensemble du territoire. En revanche, la fin du régime de J. J. Rawlings au début des années 2000 et la clôture de ce moment historique marqua un point d'arrêt de leurs activités. Avec l'alternance et l'arrivée de John Kufuor, la loi de 1992 qui autorisait les Guards à contrôler les véhicules commerciaux hors des gares et couplait le prélèvement de l'impôt sur les activités des chauffeurs commerciaux à la collecte du syndicat fut abrogée. Le prélèvement de l'impôt incombant désormais à des représentants du Trésor placés dans les gares, les revenus de l'échelon national du syndicat baissèrent drastiquement, ce qui entraîna la fin du financement de la partie du contingent de Guards qui était rattachée l'échelon national.

Ce changement dans la loi a instauré une situation contrastée. Selon un ancien formateur des Union Guards, dans la région d'Accra, une partie des Guards du contingent national a intégré l'armée ou la police ${ }^{54}$. L'étaticité incorporée a ainsi facilité la désagrégation de

\footnotetext{
${ }^{53}$ T. Bierschenk, J.-P. Olivier de Sardan (eds), States at Work: Dynamics of African Bureaucracies, op. cit., ou encore dans le contexte français, Vincent Dubois, La vie au guichet. Relation administrative et traitement de la misère, Paris, Économica, 2008.

${ }^{54}$ Les carrières militantes des Union Guards suivent ici une trajectoire bien connue des mouvements politiques violents en Afrique de l'Ouest. Richard Banégas, «La politique du "gbonhi”. Mobilisations patriotiques,
} 
l'institution qu'elle contribuait à légitimer en autorisant des carrières à cheval entre les Union Guards et les forces de l'ordre. Les Guards rattachés aux branches locales du syndicat, eux, sont restés en partie actifs. Certains ont délaissé l'uniforme pour redevenir de simples rabatteurs de clients, toujours dotés d'un prestige plus important au sein des gares en raison de leur passé de Guards. D'autres ont conservé leur uniforme et sont devenus de simples agents en charge de la sécurité et de la circulation au sein des gares et dans leurs alentours immédiats. La décadence de l'institution se lit cependant sur leurs mises, en partie dépareillées, lorsqu'elles ne sont pas rapiécées.

À l'inverse, dans la région Ashanti, où l'initiative a originellement commencé dès les années 1970, le contingent régional des Guards est resté actif. Au moment de mon enquête, en 2013, ils assuraient la sécurité aux alentours immédiats de la gare ceinturant l'immeuble où se trouvaient les locaux de la représentation régionale du GPRTU. Ils avaient aussi à leur charge l'intendance dans les bureaux des cadres du syndicat aux étages. Ils continuaient de plus d'être associés aux contrôles routiers en dehors des gares à différents endroits de la ville, sous la tutelle directe de la police municipale à qui ils servaient de force d'appoint pour la fluidification de la circulation. Cependant, cette coopération s'effectuait désormais en dehors de tout cadre légal, sans qu'il soit question d'amende ou de prélèvement d'impôt.

\section{La survie d'une autre (Sénégal)}

Les barrages organisés par les Brigades maraudage sont, eux aussi, des dispositifs fragiles. Comme pour les Union Guards, cette fragilité appelle deux remarques. Premièrement, l'étaticité bricolée laisse place au même type de transactions que l'étaticité incorporée. La socialisation des membres des Brigades à la profession de chauffeurs les expose tout autant que les Union Guards à des doubles injonctions lorsqu'il s'agit de mettre à l'amende un collègue. Cependant, n'étant pas directement encadrée par la loi, l'activité des Brigades repose sur une série de transactions informelles avec les forces de l'ordre. Cette situation les expose à la dénonciation ${ }^{55}$, risque qui n'est pas sans avoir un effet sur leurs relations aux contrevenants. En effet, la sanction pour maraudage ne varie pas seulement en fonction de la gravité de l'infraction estimée par les membres de la Brigade, mais aussi, voire surtout, en fonction du rapport entre cette estimation et le risque éventuel que le chauffeur pris en défaut dénonce la pratique des Brigades. Selon mes observations, la simple possibilité que cette pratique soit dénoncée devant une autorité ne faisant pas partie du réseau de consolidation formé par les regroupements et les forces de l'ordre - un magistrat par exemple - suffit à mettre fin à la coopération qui est à l'origine de la mise en scène de cette institution.

La façon d'argumenter, la capacité à mobiliser les bons registres, les ressources mises en avant par le chauffeur lors de la négociation systématique de l'amende peuvent tantôt suspendre celle-ci, tantôt la faire monter à son niveau le plus élevé. Or plus une transaction engage de petites sommes, moins elle suscitera de contestation. Les membres des Brigades maraudage préfèrent donc récolter des piécettes plutôt que les montants prévus pouvant

\footnotetext{
violence milicienne et carrières militantes en Côte-d'Ivoire », Genèses, 81 (4), 2010, p. 25-44.

${ }^{55}$ M. Dobry, « Valeurs, croyances et transactions collusives. Notes pour une réorientation de l'analyse de la légitimation des systèmes démocratiques », dans Javier Santiso (dir.), À la recherche de la démocratie. Mélanges offerts à Guy Hermet, Paris, Karthala, 2002, p. 112.
} 
aller de 2500 à 50000 FCFA selon le nombre de récidives ou le nombre de passagers «puisés ${ }^{56}$ sur le bord des routes. Tenter de faire appliquer le règlement à la lettre exposerait au risque de se faire rappeler son véritable statut, autrement dit de perdre la face. Au-delà des ressources dont dispose le contrevenant, ces variations du montant des amendes dépendent aussi de la place qu'occupent les regroupements dans l'espace syndical. Dans les gares les plus petites, les revenus liés à la lutte contre le maraudage occupent une place plus importante que les prélèvements opérés sur les véhicules utilisant effectivement la gare. La lutte contre le maraudage contribue en outre à l'affirmation de la gare, de son regroupement et plus largement de la ville comme une étape importante sur la route. Ces différences en engendrent d'autres, qui concernent notamment la précision du relevé des amendes et la bonne tenue des carnets. Selon mes observations, ces aspects prennent une plus grande importance dans les regroupements plus petits, où l'accent mis sur le respect de l'esthétique bureaucratique vient compenser le manque de notoriété du regroupement au sein de la profession.

Le pendant de cette variété de situations et des accords locaux informels sur lesquels elles se fondent est la relative plasticité du dispositif face aux évolutions de la conjoncture politique nationale. La fin de l'ère socialiste, en 2000, puis le changement de majorité, en 2012, n'ont eu aucune influence sur l'existence des Brigades. Les réformes qui ont touché le transport urbain dans la région de Dakar depuis la fin des années 1990 ont fait l'objet de réappropriations politiques ${ }^{57}$, mais n'ont pas affecté les Brigades qui interviennent en zones interurbaines et dans les régions. Certes, le développement des « cars horaires » prenant leur départ en dehors des gares depuis les années 2010 a mis à mal le monopole des regroupements sur la gestion du trafic de passagers, mais il n'a pas mis fin à la présence des Brigades sur le bord des routes.

Sous cet angle, la configuration d'acteurs locaux permettant l'existence des Brigades maraudage et le style d'étaticité qu'elle induit semblent plus pérennes que pour les Union Guards. Les dénonciations qui menacent éventuellement les Brigades restent confinées à l'endroit de l'interaction entre leurs membres et les contrevenants. Le rôle joué par le droit dans la légitimation des activités des Union Guards limite la possibilité de ces (micro)dénonciations. Cependant, il rend également les collusions entre le syndicat du transport et le régime plus apparentes et donc expose cette activité à des remises en cause plus radicales.

Dans cet article, la comparaison a permis de dégager deux manières de faire officiel. Celle des Union Guards du Ghana est le produit d'une série d'accords passés à l'échelle nationale et de la cristallisation par le droit du chevauchement des univers partisans, administratifs et syndicaux propres au moment révolutionnaire. Elle confère à cette institution des ressources puissantes pour mettre en scène l'officialité de ses pratiques, mais la rend sujette

\footnotetext{
${ }^{56}$ Selon l'expression consacrée dans le milieu du transport.

${ }^{57}$ Sidy Cissokho, «Réformer en situation de "décharge" : les transports publics à Dakar durant les mandats d'Abdoulaye Wade », Politique africaine, 126 (2), 2012, p. 163-184.
} 
à des dénonciations qui remettent radicalement en cause son existence. Celle des Brigades maraudage du Sénégal est le produit d'une série d'accords passés à l'échelle régionale ou municipale en dehors du droit. Elle confère à cette institution des ressources moindres pour mettre en scène son officialité et la rend en outre plus sujette à des contestations ponctuelles. Cependant, le caractère local des arrangements dont ses pratiques sont le produit la protège de toute remise en cause radicale à l'échelle nationale.

La comparaison de ces deux types offre donc deux prolongements aux débats sur l'État en Afrique. Elle permet tout d'abord d'avancer l'idée selon laquelle, lorsqu'on parle d'étaticité, le « faire croire » renvoie de façon contre-intuitive à des situations plus stables que l'incarnation. La capacité des Union Guards à incarner l'État est le produit des suites d'un coup d'État et donc d'une situation éminemment incertaine. Elle a par ailleurs été remise en cause par la clôture de cette séquence historique. En revanche, la capacité des Brigades maraudage à « faire croire » est le résultat d'échanges routiniers, dont l'existence échappe largement aux contingences historiques nationales.

Ensuite, au-delà de cette différence, l'exemple des Union Guards et des Brigades maraudage montre comment les représentations associées à l'État imprègnent la société bien au-delà de l'administration. Loin d'être concurrent de l'administration, le registre suivant lequel agissent ces deux institutions lui est étonnamment similaire. Ces deux types de barrages ne procèdent donc pas d'un manque d'État ou d'une déficience de ce dernier, mais du débordement de l'imagerie et de l'imaginaire étatiques sur un ensemble d'institutions ne disposant pas de son label. Dans ces deux cas, la référence à l'État est utilisée sur un registre légitimant - parmi d'autres - pour faire respecter des règles propres à un univers professionnel et garantir aux instances syndicales le monopole de la représentation de ce groupe, ainsi que la lucrative gestion des gares routières. C'est probablement sur ce point que se joue la principale différence entre ces institutions et le reste de l'administration. Malgré l'emploi d'une série de références à cette dernière, les Union Guards et les Brigades abandonnent à la puissance publique un élément clé de son discours : l'intérêt général. Leurs actions s'effectuent en effet avant tout au nom de normes et d'intérêts qui ne relèvent que d'un segment restreint de la société ${ }^{58}$.

\footnotetext{
${ }^{58}$ Ce texte a été rédigé dans le cadre du projet « African Governance and Space: Transport Corridors, Border Towns and Port Cities in Transition (AFRIGOS) » financé par le Conseil européen de la recherche (ERC) (ADG-2014-670851). Il se fonde cependant sur un ensemble de données recueillies lors de terrains préalables. Je remercie tout particulièrement Paul Nugent pour ses conseils précieux sur une version antérieure de cet article, ainsi que Anne Hugon et Antoine de Boyer pour leur aide précieuse avant et pendant mon terrain ghanéen.
} 